[0212-7199 (2003) 20: 12; pp 630-632]
ANALES DE MEDICINA INTERNA ANALES DE MEDICINA INTERNA Copyright $\odot 2003$ ARAN EDICIONES, S.L.

AN Med INTERNA (Madrid) Vol. 20, N. ${ }^{\circ} 12$, pp. 630-632, 2003

\title{
Sepsis por Vibrio cholerae no-01 en paciente inmunodeprimida
}

\author{
J. V. CALDUCH BROSETA, M. M. SEGARRA SORIA, J. COLOMINA AVILÉS, \\ C. LLORCA FERRANDIZ1', R. PASCUAL PÉREZ
}

Servicio de Medicina Interna. ${ }^{~}$ Sección de Oncología. Hospital General de Elda. Alicante

\author{
SEPTICEMIA CAUSED BY VIBRIO CHOLERAE NON-01 IN IMMU- \\ NOCOMPROMISED PATIENT
}

\begin{abstract}
RESUMEN
Describimos el caso poco frecuente de una paciente con una sepsis por Vibrio Cholerae no 0-1 asociado a lesiones cutáneas en extremidades inferiores y superiores. Esta paciente padecía de una hepatopatía crónica y un carcinoma de cérvix en estadío IIIB y había sido ingresada el día previo por una descompensación ascítica. Su evolución fue buena con resolución completa mediante cefotaxima endovenosa. Destacar sobre todo el hallazgo de su origen epidemiológico, aguas continentales de pozos naturales, siendo esta particularidad la que hace de este caso el primero descrito en España con ese origen demostrado y un caso excepcional en la literatura mundial revisada.
\end{abstract}

PALABRAS CLAVE: Bacteriemia. Vibrio Cholerae no-01. Cirrosis.

\begin{abstract}
We describe the remarkable case of a patient with septicemia caused by Non 0-1 Vibrio Cholerae associated with skin lesion of the lower and upper extremities. This patient suffered from chronic liver disease and a cervix carcinoma in IIIB stage, she had been admitted to the hospital the day before for dicompensated ascites. She received intravenous cefotaxime and had a satisfactory recovery and was completely free of signs and symptoms. We report its epidemiological discovery in inland freshwater and this is the first announced case in Spain with this confirmed environmental isolation and a rare report case in the literature.
\end{abstract}

KEY WORDS: Bacteremia. Vibrio Cholerae non-01. Cirrhosis.

Calduch Broseta JV, Segarra Soria MM, Colomina Avilés J, Llorca Ferrandiz C, Pascual Pérez. R. Sepsis por Vibrio cholerae no-01 en paciente inmunodeprimida. An Med Interna (Madrid) 2003; 20: 630-632.

\section{INTRODUCCIÓN}

El Vibrio cholerae no-01 (VCN-01), es una bacteria gramnegativa que no se aglutina en presencia de suero específico (vibrios no aglutinables o NAG) $(1,2)$. Producen su efecto por medio de factores tóxicos múltiples (enterotoxinas y exotoxinas) (3). Tiene una amplia distribución mundial, estudios medioambientales han demostrado que suelen hallarse en entornos marinos, bahías y estuarios donde la salinidad de las aguas oscila de $0,4 \%-1,7 \%$ con una temperatura mayor de $20^{\circ} \mathrm{C}$ $(4,5)$, comunicándose por esto la mayor parte de casos durante los meses de calor (6). La principal fuente de trasmisión es la ingesta de alimentos de origen marino, cangrejos y ostras consumidos crudos, y también la exposición directa de lesiones en la piel al agua de mar (5-7). Las manifestaciones clínicas que puede dar lugar son intestinales como gastroenteritis, y extraintestinales, como otitis media, celulitis, infecciones del tracto biliar y raras veces bacteriemias $(1,2,6,8-10)$. Estas últi- mas se han descrito asociadas a factores predisponentes como cirrosis hepática y enfermedades malignas hematológicas $(7,11,12)$.

Describimos un caso de sepsis y lesiones cutáneas en una paciente con una hepatopatía crónica y un carcinoma de cérvix avanzado.

\section{CASO APORTADO}

Una mujer de 78 años fue ingresada en nuestro hospital en agosto, a causa de un cuadro de deterioro general con descompensación hidrópica. Había sido diagnosticada de un carcinoma de cérvix en estadio IIIB y tratada mediante radioterapia, desarrollando posteriormente una proctosigmoiditis postradioterapia que periódicamente le causaba ligeras rectorragias, para lo que recibía enemas con esteroides. Además, también estaba diagnosticada de una cirrosis hepática de origen no filiado. Vivía en una casa rural, donde cuidaba animales de granja. Disponían de agua desde un pozo natural y agua potable

Trabajo aceptado: 22 de abril de 2003

Correspondencia: J. V. Calduch Broseta. C/ Alcalde Alfonso de Rojas, 2, 2º A. 03004 Alicante. e-mail: JVCAL_06@terra.es 
que traían en garrafas desde otra finca. Negaba hábitos tóxicos y viajes al extranjero y decía ocasionalmente consumir carnes de animales que criaba.

En la exploración física la $\mathrm{T}^{\mathrm{a}}$ era de $36,7{ }^{\circ} \mathrm{C}$, la TA $120 / 70$ $\mathrm{mmHg}$ y el pulso de $74 \mathrm{lpm}$. La enferma se encontraba consciente y orientada, mostrando estigmas de hepatopatía crónica, palidez de piel y mucosas, un abdomen con ascitis evidente sin signos de peritonismo y edemas bimaleolares con trastornos tróficos cutáneos crónicos en ambas piernas.

En la analítica destacaba una hemoglobina de $6,8 \mathrm{~g} / \mathrm{dl}$, un hematocrito de $21,4 \%$, un VCM de $75 \mathrm{fl}$, unos leucocitos de 5.100 $/ \mathrm{mm}^{3}$ sin desviación izquierda, una creatinina de $1,8 \mathrm{mg} / \mathrm{dl}$, un sodio de $131 \mathrm{mE} / \mathrm{l}$, un hierro de $15 \mathrm{mg} / \mathrm{dl}$, un índice de Quick de 59\%, siendo el resto de los parámetros de la bioquímica y coagulación normales. El sedimento de orina fue anodino y su cultivo negativo. A su ingreso se realizó una paracentesis diagnóstica que reflejó que se trataba de un trasudado con cultivo negativo. Se decidió trasfundirle sangre ese mismo día para mejorarle de su anemia y a las 12 horas presentó un cuadro brusco de malestar general, tiritonas con fiebre de $39{ }^{\circ} \mathrm{C}$, hipotensión de $85 / 50 \mathrm{mmHg}$, obnubilación y aparición de lesiones cutáneas petequiales, confluentes en EEII y posteriormente en EESS, las cuales evolucionaron hacia la formación de ampollas con contenido serohemático.

Con la sospecha de síndrome séptico, se obtuvieron 3 hemocultivos iniciándose tratamiento con volumen y antibioterapia empírica con cefotaxima $1 \mathrm{~g}$ i.v. cada 6 horas que se prolongó durante 14 días, así como limpieza de las ampollas y tratamiento tópico con tetraciclina. La evolución fue satisfactoria desapareciendo la fiebre en las siguientes 48 horas y mejorando lentamente las lesiones cutáneas.

Días más tarde, se recibían los resultados de los hemocultivos: Vibrio cholerae no-01 (3/3), que fueron además contrastados por la Sección de Enterobacterias Centro Nacional de Microbiología y Virología de Majadahonda, sensibles al tratamiento administrado (también a quinolonas y a tetraciclinas). Esta cepa no se aisló de los coprocultivos a pesar de que incluyeron medios TCBS, probablemente porque había recibido antibioterapia en los 3 días previos a su recogida.

TABLA I

ESTUDIO BIOLÓGICO/BIOQUÍMICO DE LAS AGUAS

\begin{tabular}{lcc}
\hline & $A$ & $B$ \\
\hline Nitritos ml/l & $<0,05$ & $<0,05$ \\
Amoniacos mg/l & 0,18 & 0,05 \\
Cloro residual mg/l & $\mathrm{ND}$ & $\mathrm{ND}$ \\
Nitratos mg/l & $<0,5$ & 6,54 \\
Sulfatos mg/l & 15556,5 & 81,1 \\
Dureza $\mathrm{mg} / \mathrm{l}$ & 718,4 & 142,4 \\
pH & 7,44 & 8,4 \\
Oxidabilidad & 2,86 & $<0,9$ \\
Cloruros mg/l & 1030 & 144 \\
Calcio mg/l & 333 & 56 \\
Flúor mg/l & 2729,4 & 551,4 \\
Bacterias aerobias $37^{\circ}$ & 33 & 1100 \\
Bacterias aerobias $22^{\circ}$ & 23 & 540 \\
Coliformes totales/100 ml & 100 & Incontables \\
Coliformes fecales/100 ml & 440 & 0,0 \\
Estreptococos fecales/10 ml & 440 & 0,0 \\
Clostridium sulfito- reductores/20 ml & 25 & 4 \\
Salmonella 100 ml & Ausencia & Ausencia \\
Vibrio cholerae no-01 & Presencia & Presencia \\
\hline
\end{tabular}

$\mathrm{A}=$ Fuente natural, $\mathrm{B}=$ Agua traída para su condumo (desde otros pozos naturales próximos)
Nuestra paciente vivía en una casa de campo. El agua del manantial que emerge del subsuelo a una balsa descubierta era distribuida mediante una serie de gomas para riego, para el ganado y al interior de la vivienda donde era utilizada para el lavado de utensilios y aseo personal y que el agua para su consumo se traía de otra finca. Avisado al Centro de Salud Pública local se inició la investigación pertinente, procediendo a tomar muestras de las aguas y obteniendo los siguientes resultados (Tabla I): presencia de Vibrio cholerae no-01 tanto en el agua del pozo usado para riego, cuidado de animales y aseo como del agua utilizada para consumo humano, confirmándose estos hallazgos también en el Centro nacional de Microbiología, destacando la mala calidad de las aguas usadas para el consumo humano, con una tasa elevada de contaminación biológica de las muestras obtenidas, así como con una salinidad mayor de lo habitual.

\section{DISCUSIÓN}

La manifestación clínica más frecuente del VCN-01 es la diarrea bien como casos aislados o como brotes epidémicos, y su espectro abarca desde la diarrea leve hasta una severa diarrea acuosa indistinguible de la clásica diarrea colérica (13). Son frecuentes el dolor abdominal $(9,13,14)$, las náuseas y vómitos, $\mathrm{y}$ es variable la presencia de fiebre, pero en alguna serie llega a detectarse en el 43-71\% de los enfermos, signo raro en el cólera clásico $(2,9,13)$. Ocasionalmente, produce diarreas con sangre (13), hecho atribuido a la producción de toxinas disenteriformes o a poseer capacidad invasiva (1). La recuperación suele ser la norma, incluso sin el empleo de antimicrobianos (14) aunque se recomiendan en los casos severos, pues disminuyen la duración y severidad del cólera (6). Aun siendo el principal reservorio del VCN-O1 el ambiente acuático de las aguas costeras, éste se ha aislado en ostras frescas cultivadas, sobretodo, en los meses de verano (9), cangrejos y camarones (6). Se ha descrito en seres humanos el estado de portador asintomático (9), que en un estudio fue casi del 4\% (15).

Aunque las bacteriemias por este germen son excepcionales, representan el segundo aislamiento más frecuente $(6,14)$ y las series más numerosas se han publicado en los últimos años provenientes del continente asiático en pacientes cirróticos, en zonas endémicas del virus de la hepatitis B $(7,14)$. En España, en los últimos 20 años sólo hemos recogido publicados 17 casos $(11,16,17)$ y de éstos son muy pocos los que se han asociado a lesiones cutáneas $(11,16,18)$. En la infección de piel y partes blandas puede haber antecedente de heridas o pequeños traumatismos y contacto con agua salada o dulce $(14,19,20)$ o producirse en el curso de una bacteriemia (14). En esta celulitis lo más característico es la formación de bullas hemorrágicas en las extremidades inferiores (16), como en nuestro caso. La presencia de diarrea es bastante variable, pero sí que es raro aislar los vibrios en heces $(1,11)$, probablemente porque ya reciben antibióticos cuando se recogen las heces, como en nuestra enferma, o porque no se empleen medios especiales (TCBS) de manera rutinaria (1) o por no ser esta la puerta de entrada. La ausencia de antecedentes epidemiológicos clásicos (ingesta de pescado o marisco crudos o poco cocinados y exposición a aguas saladas) también es frecuente en nuestros casos. A pesar de que otros autores asocian un pronóstico peor a la bacteriemia con celulitis (7), los casos españoles sobrevivieron sin drenaje local $(11,16)$. Posiblemente un desbridamiento extenso se asocie a mejor pronóstico (7) o la aspiración de las bullas (21) en algunos pacientes. En las series asiáticas $(7,14)$ la mortalidad $(28-47 \%)$ es menor que en la 
revisión de Safrin et al (12) que era del 61,5\%, pero superior a la mortalidad del $16,6 \%$ de los casos españoles. Cuando Fernández et al revisan la mortalidad asociada de 17 casos en la literatura médica anglohispana de celulitis bacteriémica por VCN-01 la mortalidad global era del 50\% (16). En cuanto a su tratamiento, se han usado ampicilina, tetraciclina, cefalosporinas, aminoglucósidos y quinolonas $(7,12,14,16,21)$ con éxito. Aunque se ha recomendado el uso de la combinación de tetraciclinas y aminoglucósidos (11), parece más recomendable combinar cefotaxima y doxiciclina o una nueva fluoroquinolona (14). En nuestra enferma, la desaparición de la fiebre en 48 horas y la progresiva mejoría del estado general hicieron que no modificáramos el tratamiento iniciado empíricamente.

El caso aquí presentado es similar a otros comunicados en series más amplias $(7,11,14,16,19)$, lo que pensamos que le hace más peculiar es su foco de contagio. De la historia clínica se sabía que la paciente no había ingerido alimentos de origen marino en los días previos y que no había paseado por la orilla del mar desde hacía varios años. Estos son los antecedentes epidemiológicos relacionados con el Vibrio cholerae no-01 $(1,2,6)$.

Aun siendo estos vibrios patógenos la flora bacteriana autóctona de ambientes marinos y estuarios, también se han aislado en aguas de ríos (22), lagos $(19,20)$, pozos (17), aguas residuales (23).
En la revisión realizada, se trata del primer caso descrito en nuestro país con este foco epidemiológico demostrado. La puerta de entrada pudo ser tanto la vía oral, mediante el agua de consumo y de aseo, como a través de la piel de las piernas, pues presentaba lesiones derivadas de sus problemas de insuficiencia venosa.

Aunque no todas las cepas de Vibrio cholerae son capaces de colonizar el tracto gastrointestinal o causar diarrea (3), nuestra enferma ingería a diario agua de consumo contaminada y presentaba unas lesiones inflamatorias de la mucosa intestinal derivadas de la radioterapia que trataba con esteroides que en cualquier caso facilitarían su paso por vía hemática. En resumen, la bacteriemia por Vibrio cholerae no-01 aunque poco frecuente, puede ser una grave complicación en pacientes con cirrosis o enfermedades hematológicas malignas. En estos enfermos debería de avisarse que evitaran la ingesta de alimentos marinos como mariscos y cangrejos y que pasearan por el mar si presentan lesiones cutáneas. Por otro lado y a la vista de los resultados obtenidos, considerar también las aguas interiores como posible foco de la enfermedad al ingresar en la cadena alimentaria, así como a estimular a las autoridades sanitarias, a la vista de su descubrimiento en aguas de pozos naturales usados para el consumo humano, a un más exhaustivo control de nuestros acuíferos y de la red de distribución de aguas.

\section{Bibliografía}

1. Prats G. Infecciones causadas por Vibrio Cholerae no-01. Enf Infect Microbiol Clín 1987; 2: 577-581.

2. Hugues JM, Hollis DG, Gangarosa EJ, Weaver RE. Non-Cholera vibrio infections in the United States. Clinical, epidemiologic and laboratory features. Ann Intern Med 1978; 88: 602-606.

3. Panigraphi P, Tall BD, Russell RG, Detolla LJ, Morris JG Jr. Development of an in vitro model for study of Non-01 Vibrio cholerae virulence using Caco-2 cells. Infect Inmun 1990; 58: 3415-24.

4. Oliver JD, Warner RA, Cleland DR. Distribution and ecology of Vibrio vulnificus and other lactose-fermenting marine vibrios in coastal waters of the southeastern United States. Appl Environ Microbiol 1982; 44: 1404-14.

5. De Paola A. Vibrio cholerae in Marine Foods and environmental Waters: A literature review. J Food Sci 1981; 46: 66-70.

6. Morris JG, Black RE. Cholera and other vibrioses in the United States. New Engl J Med 1985; 312: 343-50.

7. Lin Ch-J, Chiu Ch-T, Lin D-Y, Sheen I-Sh, Lien J-M. Non-01 Vibrio cholerae becteremia in patients with cirrosis: 5-yr experience from a single medical center. AJG 1996; 91: 336-40.

8. Forné M, Matas E, Martí C, Pujol R, Garau J. Sepsis por Vibrio cholerae no-01. Enf Infect Microbiol Clin 1987; 2: 590-594.

9. Morris JG Jr. Non-0 group 1 Vibrio cholerae : a look at the epidmiology of an occasional pathogen. Epidemiol Rev 1990; 12: 179-91.

10. Blake PA. Diseases of humans (other than cholera) caused by vibrios. Ann Rev Microbiol 1980. 34: 341-67.

11. Catalá MT, Núñez JC, Balagur JV, Borrás R. Sepsis por Vibrio cholerae no -01 en paciente sano: revisión de los casos publicados en España. Rev Clín Esp 1998; 198 (2): 850-51.

12. Safrin S, Morris JG Jr, Adams M, Pons V, Jacobs R, Conte JE Jr. Non01 Vibrio cholerae bacteremia. Case report and review. Rev Infect Dis 1988; 10:1012-17

13. Morris JG Jr., Wilson R, Davis BR, et al. Non-0 group 1 Vibrio cholerae

gastroenteritis in the United States. Clinical, epidemiologic and laboratory characteristics of sporadic cases. Ann Intern Med 1981; 94: 656-8.

14. Ko W-Ch, Chuang Y-Ch, Huang G-Ch, Hsu Sh-Y. Infections due to Non-01 Vibrio cholerae in Southern Taiwan: Predominance in cirrotic patients. Clin Infect Dis 1998; 27: 774-80.

15. Lowry PW, Mcfarland LM, peltier $\mathrm{BH}$ et al. Vibrio gastroenteritis in Louisiana: a prospective study among attendees of a scientific congress in New Orleans. J Infect Dis 1989; 160: 978-984.

16. Fernández JM, Serrano M, De Arriba JJ et al. Bacteremic cellulitis caused by Non-01, Non-0139 Vibrio cholerae: report of a case in patient with hemochromatosis. Diagn Microbiol Infect Dis 2000; 37: 77-80.

17. Esparcia AM, Cañizares R, Roig P, Martínez A. Bacteriemia por Vibrio cholerae no 01. A propósito de dos casos. Enf Infect Microbiol Clin 2000; 18: 65-66

18. Royo G, Martín C, Fuentes E, Elía M, Fernández J, Cuesta A. Bacteriemia por Vibrio cholerae no-01. Enf Infect Microbiol Clin 1993; 11 (4): 228

19. Mulder GD, Reis TM, Beaver TR. Nontoxigenic Vibrio cholerae wound infectionafter exposure to contamined lake water. J Infect Dis 1989; 159: 809-811.

20. Pitrak DL, Gindorf JD. Bacteremic cellulites caused by non-sero-group 01 Vibrio cholerae acquired in a freshwater inland lake. J Clin Microbiol 1989;L 27: 2874-6.

21. Kontoyiannis DP, Calia KE, Basgoz N, Calderwood SB. Primary septicemia caused by Vibrio cholerae Non-01 acquired on Cape Cod, Massachusetts. Clin Infect Dis 1995; 21: 1330-3.

22. Rhodes JB, Smith HL Jr, Ogg JE. Isolation of non-01 Vibrio cholerae serovars from surface waters in Western Colorado. Appl Environ Microbiol 1986; 51: 1216-1219.

23. Radu S, Ho YK, Lihuan S, et al. Molecular characterization of Vibrio cholerae 01 and non-01 from human and environmental sources in Malaysia. Epidemiol Infect 1999; 123: 225-232. 